\title{
AMELANOTIC MELANOMA WITH ATYPICAL CLINICAL PRESENTATION AND MULTIPLE METASTASIS
}

Revathy Mathan' ${ }^{1}$ Ramasamy P. P2

\section{HOW TO CITE THIS ARTICLE:}

Revathy Mathan, Ramasamy P. P. "Amelanotic Melanoma with Atypical Clinical Presentation and Multiple Metastasis". Journal of Evolution of Medical and Dental Sciences 2014; Vol. 3, Issue 65, November 27;

Page: 14230-14234, DOI: $10.14260 /$ jemds/2014/3907

ABSTRACT: A 52 year old woman presented with a history of asymptomatic skin lesions over left leg for the past 4 months. On examination she had multiple skin coloured papules and plaques over left leg. Oedema was also seen over left leg. Histopathology and immunohistochemistry proved the diagnosis of malignant melanoma. Radiological investigation showed metastasis to lung, liver and brain. The patient was asymptomatic at the time of admission but she developed rapid metastasis within a very short span of time. This case is reported for the rare atypical presentation of malignant melanoma.

KEYWORDS: malignant melanoma, amelanotic, metastasis.

INTRODUCTION: Malignant melanoma is a neoplasm of melanocytes or a neoplasm of the cells that develop from melanocytes. It accounts for $1-3 \%$ of all malignancies and $5 \%$ of all skin malignancies. It most commonly spreads by lymphatics with a rapid systemic dissemination. Common site of spread is lymphnode and lung followed by liver, brain and adrenals. Melanomas lacking clinically evident pigment are termed amelanotic. Here, we report a case of amelanotic melanoma with atypical clinical presentation and multiple metastases.

CASE REPORT: 52 year old female patient presented with a history of asymptomatic skin lesions and diffuse swelling of left leg for past 4 months. There was no history of weight loss and appetite. She is a known diabetic and has undergone amputation of $3^{\text {rd }}, 4^{\text {th }}$ and $5^{\text {th }}$ toes of left foot. Examination revealed multiple skin coloured papules with umbilication and plaques with erosions of size $0.5 \mathrm{~cm}$ over left leg (Figure: 1). Oedema of the left leg and loss of $3^{\text {rd }}, 4^{\text {thand }} 5^{\text {th }}$ toes of left foot was noted. Her left inguinal lymphnodes were enlarged.

Differential diagnoses considered were chromoblastomycosis and penicilliosis.

Haematological and biochemical parameters were normal.

Skin biopsy showed poorly circumscribed spindle cell tumours composed of irregular bundles of pleomorphic spindle cells with hyperchromatic nuclei amidst interstitial collagen Features were suggestive of malignant melanoma (Figure: 2)

Immunohistochemistry demonstrated strong S100 staining (Figure: 3 )

Vimentin staining was weakly positive.

HMB-45 staining was weakly positive. While cytokeratin was negative fine needle aspiration of a left inguinal lymphnode showed cohesive and non-cohesive clusters of plump oval to spindle shaped cells exhibiting nuclear atypia and pleomorphism.

Differential diagnosis given by the pathologist for these poorly differentiated deposits included:

1. Amelanotic melanoma.

2. Poorly differentiated squamous cell carcinoma.

3. Sarcoma. 


\section{CASE REPORT}

Based on the skin biopsy and fine needle aspiration cytology we arrived at the diagnosis of amelanotic melanoma.

USG abdomen showed secondaries liver. CT chest showed multiple lung metastases.

The patient was started on chemotherapy with vinblastine, dacarbazine in stipulated dose.

Within one week she developed right hemipaeresis. An MRI brain showed lesion in left temporal region and she underwent intracranial irradiation.

DISCUSSION: Melanoma is a malignant tumour that arises from melanocytes and is most commonly cutaneous in origin. The majority of melanomas are brown to black in colour due to melanin deposition, some are skin coloured to pink-red. Melanomas lacking clinically evident pigment are termed amelanotic.

Four major clinical subtypes of primary cutaneous melanoma have been described ${ }^{1}$ They are superficial spreading melanoma, nodular melanoma, acral lentiginous melanoma and lentigo maligna melanoma. Other variants include amelanotic melanoma, desmoplastic melanoma, spitzoid melanoma, ocular melanoma and mucosal melanoma.

In light skinned individuals risk factors include high exposure to sunlight in childhood, multiple sun burns, multiple naevi, immunodeficiency and familial predisposition. ${ }^{2}$

Histopathology remains the gold standard investigation for the diagnosis of melanoma. It has been proposed that melanomas progress through two phases. ${ }^{3}$ The radial phase is characterized by the centrifugal spread of neoplastic melanocytes within the epidermis and infiltration of papillary dermis by single cells or small nests and a vertical phase characterized by dermal nests or nodules of atypical melanocytes. Of the histological features of primary cutaneous melanoma Breslow thickness is the strongest predictor of survival.

Immunohistochemistry can be helpful in the assessment of diagnostically difficult cases of primary melanomas as well as metastatic tumours of unknown origin. TNM classification is followed in the staging of malignant melanoma. Prognosis of a patient with melanoma depends upon the stage at diagnosis.

Metastasis may manifest as a clinically occult lymph node micro metastasis, as a rapidly growing clinically evident lymphnode macrometastasis or as an in transit metastasis.

A case of amelanotic melanoma with multiple secondaries has been reported. ${ }^{4}$

The clinical and dermatoscopic features of amelanotic melanoma have been described. ${ }^{5}$

Treatment includes excision with appropriate margins determined by Breslow thickness for primary lesions and isolated metastasis. Chemotherapy and irradiation are often used without great benefit. Immunotherapy ${ }^{6}$ and BRAF inhibitors ${ }^{7}$ show great promise in treating melanoma. 


\section{CASE REPORT}

Fig. 1: Examination revealed multiple skin coloured papules with umblication and plaques with erosions of size $0.5 \mathrm{~cm}$ over left leg. Edema of the left leg and loss of $3^{\text {rd }} 4^{\text {th }}$ and $5^{\text {th }}$ toes of left foot was noted

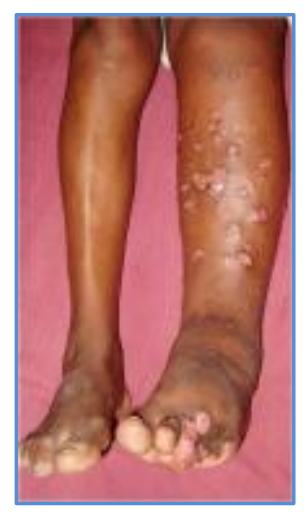

\section{Fig. 1}

Fig. 2: Poorly circumscribed tumour composed of irregular bundles of pleomorphic spindle cells with hyperchromatic nucleiadmist interstitial collagen. Features were suggestive of malignant melanoma.

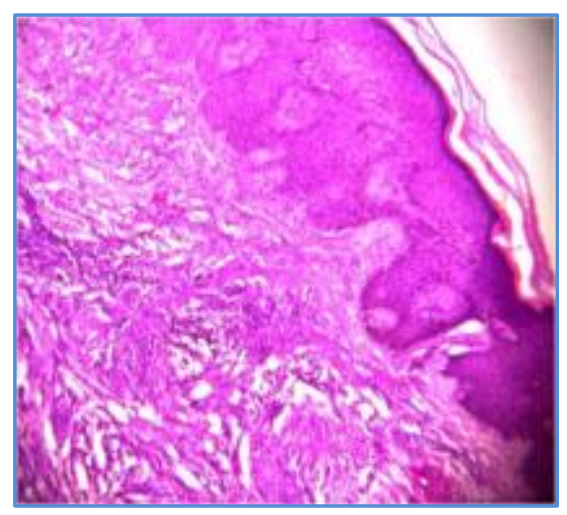

\section{Fig. 2}

Fig. 3: S100 strongly positive.

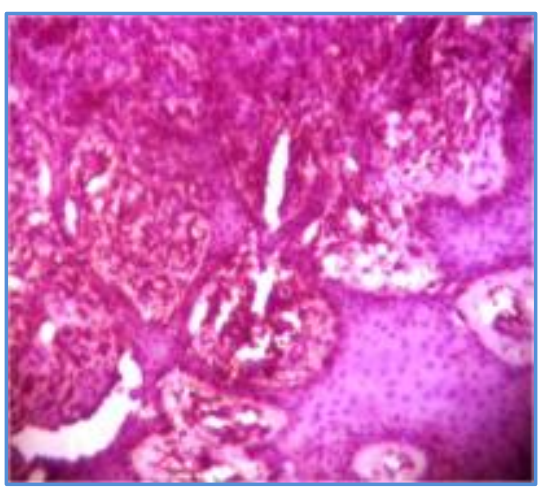

\section{Fig. 3}




\section{CASE REPORT}

Fig. 4: Vimentin weakly positive.

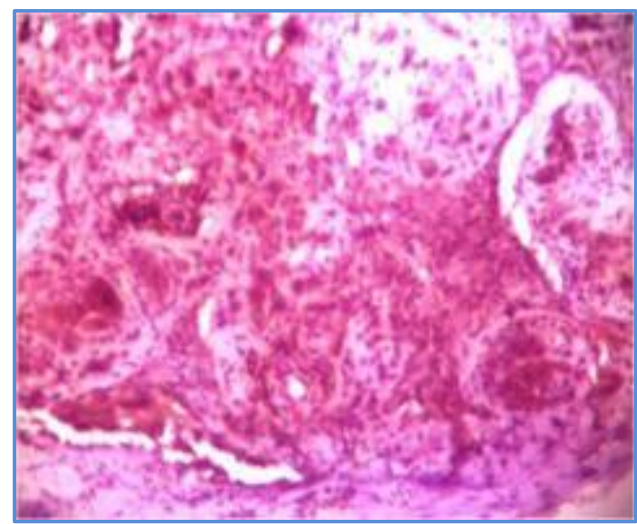

\section{Fig. 4}

Fig.5: HMB-45 weakly positive.

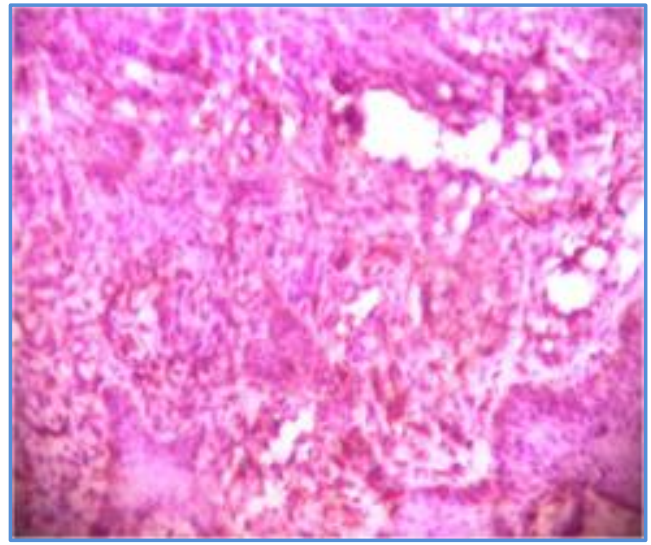

\section{Fig. 5}

Fig. 6: CT chest shows multiple lung metastasis.

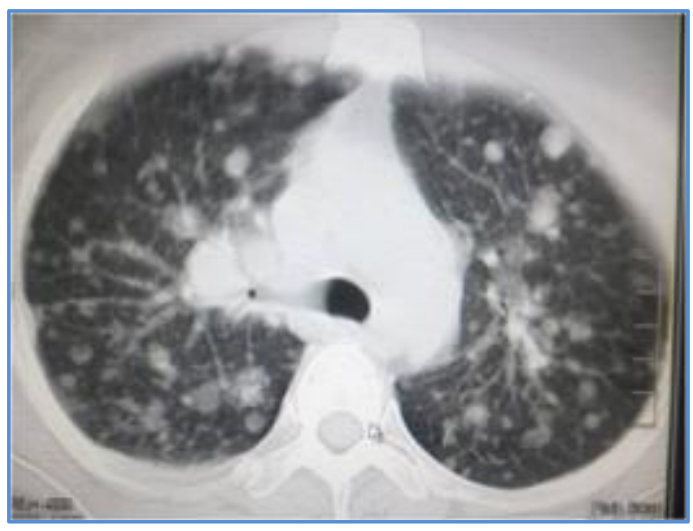

Fig. 6 


\section{CASE REPORT}

Fig. 7: MRI brain showed the lesion in left temporal region.

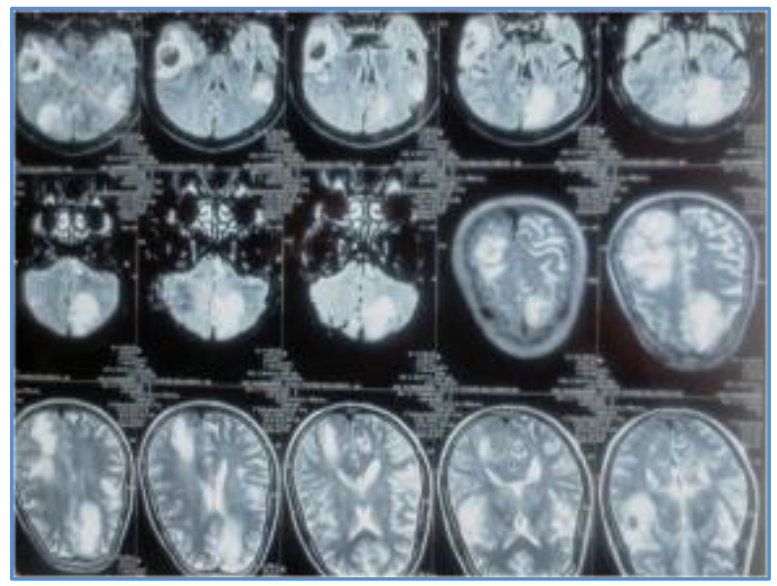

Fig. 7

\section{REFERENCES:}

1. Clark WH jr, The histogenesis and biologic behaviour of primary tumour malignant melanoma of skin, cancer Res 1969; 29; 705-27.

2. De vries E, coebergh JW, cutaneous malignant melanoma in Europe. Eur j cancer 2004; 40; 2355-66.

3. Clemente c, cook M, Ruiter D, Mihm M. Histopathologic diagnosis of melanoma. WHO program publications Milan Trezz. SN 2001.

4. Santanu Banerjee IJDVL 2013 p 115.241.111.217.

5. Pizzichetta MA BJD 2004 150: 1117-24.

6. Zeiser R, Schnitzler M, Andrlova H, Hellige T, Meiss F. Department of Hematology and Oncology, Freiburg University Medical Center, current stem cell research \& therapy volume 7: issue 3.

7. M. omar F. khan and carroll L Ramos Journal: clinical medicine insights: Dermatology 2013: 61-7.

\section{AUTHORS:}

1. Revathy Mathan

2. Ramasamy P.P

\section{PARTICULARS OF CONTRIBUTORS:}

1. Assistant Professor, Department of Dermatology, Coimbatore Medical College Hospital, Coimbatore.

2. Professor, Department of Dermatology, Coimbatore Medical College Hospital, Coimbatore.

\section{NAME ADDRESS EMAIL ID OF THE CORRESPONDING AUTHOR: \\ Revathy Mathan, Assistant Professor, Department of Dermatology, Coimbatore Medical College Hospital, Coimbatore. \\ Email: revathymithu@gmail.com}

Date of Submission: 15/11/2014. Date of Peer Review: 16/11/2014. Date of Acceptance: 24/11/2014. Date of Publishing: 27/11/2014. 